\title{
Cholinergic Deafferentation of Prefrontal Cortex Increases Sensitivity to Cross-Modal Distractors during a Sustained Attention Task
}

\author{
Lori A. Newman and Jill McGaughy \\ Department of Psychology, University of New Hampshire, Durham, New Hampshire 03824
}

\begin{abstract}
The effects of restricted cholinergic deafferentation of prefrontal cortex in rats on sustained attention were assessed. Attentional demands were increased by presentation of distractor stimuli in a different modality (auditory) or the same modality (visual) as target stimuli. Additionally, the effects of the regularity of the distractor on rats' ability to disregard this stimulus were assessed by testing different frequencies of stimuli for each modality. Cholinergically lesioned rats were more sensitive to the effects of auditory distractors than nonlesioned rats, whereas visual distractors of any frequency potently impaired the performance of all subjects. The effects of the auditory stimuli on attentional performance varied depending on the frequency of the tone. A tone with a predictable pattern enhanced signal detection in all rats. An irregular tone selectively impaired performance of rats with cholinergic lesions. Additional tests suggest that rats use the regular tone to time when to attend. Lesioned rats were impaired when the regular tone was presented with a more variable intertrial interval in a subsequent testing session, suggesting impairments in top-down control. In addition to changes in top-down control of attention, differential effects on performance based on the regularity of the tone suggest that stimulus properties encoded by bottom-up processes are also altered after lesioning. The current data suggest that cholinergic deafferentation of prefrontal cortex alters top-down and bottom-up processing of stimuli.
\end{abstract}

Key words: infralimbic cortex; prelimbic cortex; 192 IgG saporin; executive control; top-down control; selective attention

\section{Introduction}

Converging evidence supports a role for cortical acetylcholine (ACh) in many types of attention (Muir et al., 1995; McGaughy et al., 1996; Turchi and Sarter, 1997; Baxter and Chiba, 1999; McGaughy et al., 2000; Kozak et al., 2006). Much of these data are based on manipulations of the cholinergic cell bodies in the nucleus basalis magnocellularis (nbm) that alter ACh throughout the cerebral cortex (Bigl et al., 1982; Mesulam et al., 1983) and failed to dissociate the unique contributions of cortical subregions that receive cholinergic innervation. Current hypotheses suggest that the entire neocortical mantle is not functionally homogeneous; rather, distinct subregions of the cortex are recruited depending on the attentional demands (Dias et al., 1997; Mesulam, 1999; Dalley et al., 2004). One subregion, prefrontal cortex (PFC), is consistently implicated in mediating many forms of behavioral control (Mesulam, 1999; Dalley et al., 2004), and cholinergic efflux in this area is critical to these functions (Himmelheber et al., 2000; Dalley et al., 2001; McGaughy et al., 2002a; Kozak et al., 2006).

Acetylcholine in PFC is hypothesized to control top-down

Received June 29, 2007; revised Dec. 22, 2007; accepted Jan. 16, 2008.

We thank Emily Carter, Dave Creer, Jenna Darling, Dorianna Kenton, and Michelle Wasserman for excellent technical assistance in portions of the behavioral training.

Correspondence should be addressed to Jill McGaughy, Department of Psychology, University of New Hampshire, Conant Hall, 10 Library Way, Durham, NH 03824. E-mail: j.mcgaughy@unh.edu.

D01:10.1523/JNEUROSCI.5112-07.2008

Copyright $\odot 2008$ Society for Neuroscience $\quad$ 0270-6474/08/282642-09\$15.00/0 allocation of attentional resources (Sarter et al., 2001a, 2005). Increases in prefrontal ACh stimulates cholinergic efflux in the posterior parietal cortex (PPC) (Nelson et al., 2005). The parietal cortex controls spatial (Posner et al., 1987) and temporal (Coull and Nobre, 1998) orientation of attention and maintains focus in the presence of distraction (Shomstein and Yantis, 2004, 2006). The recruitment of PPC by PFC is hypothesized to result from increased attentional demand (Nelson et al., 2005).

We assessed the effects of cholinergic deafferentation of PFC in rats performing a test of sustained attention (McGaughy and Sarter, 1995a). We hypothesized that these lesions would disrupt top-down control of attention necessary to disregard distractors. Previous work has shown that prefrontal cortical neurons fire in response to distractors (Gill et al., 2000; Buschman and Miller, 2007). Cholinergic deafferentation of PFC decreased the firing rate of prefrontal neurons and attenuated "distractor-induced positive modulation of neuronal responses," supporting the hypothesis that cortical acetylcholine is necessary to disregard irrelevant stimuli (Gill et al., 2000). Because visual distractors potently disrupt performance of intact rats and produce floor effects in behavior (McGaughy and Sarter, 1995a), we also assessed the effects of auditory distractors. We hypothesized that cholinergically lesioned (ACh-LX) rats would be unable to disregard all task-irrelevant stimuli. Additionally, we varied the duration of on-off cycles of distractors to determine whether increased similarity between visual distractors and targets exacerbated attentional impairments. The effects of auditory distractors were not 
expected to differ based on the on-off pattern of the stimuli because these stimuli had no perceptual overlap with targets. To assess the effects of other types of increased attentional demands, we tested subjects with a more unpredictable event rate (Scerbo et al., 1987; McGaughy and Sarter, 1995a). Finally, we assessed the effects of eliminating the dynamic stimulus range (Parasuraman et al., 1987) to determine whether cholinergic lesions influence the response to decreases in expected uncertainty ( $\mathrm{Yu}$ and Dayan, 2002, 2005).

\section{Materials and Methods \\ Subjects}

Sixteen male, Long-Evans rats (Harlan, Indianapolis, IN) were used in this study. The animals were moderately food restricted to keep their weights at $90 \%$ of age-matched controls receiving $\sim 18 \mathrm{~g}$ of rat chow per diem and ad libitum water. All animals were weighed weekly to ensure healthy weights relative to age-matched norms. Animals were housed separately, kept on a $12 \mathrm{~h}$ light/dark cycle (lights on at 6:00 A.M.) in a climate-controlled environment, and only tested during the light hours.

\section{Apparatus and materials}

Operant chambers (Med Associates, St. Albans, VT) equipped with two retractable levers, a house light $(2.8 \mathrm{~W})$, a $45 \mathrm{mg}$ pellet dispenser, a 2900 $\mathrm{Hz}$ sonalert tone generator, and three panel lights $(2.8 \mathrm{~W})$ were used. The food dispenser, panel lights, and retractable levers were all located on the same wall. The tone generator and house light were located on the opposite wall. Records of signal presentation, lever operation, and food pellet (Noyes Precision Pellets, 45 mg; Research Diets, New Brunswick, NJ) delivery were maintained using a personal computer with Windows XP (Microsoft, Seattle, WA) and the Med-PC IV software (Med Associates).

\section{Behavioral training}

Training occurred between 8:00 A.M. and 2:00 P.M. 5 d/week. Rats were initially trained to bar press for food in accordance with a fixed ratio 1 schedule of reinforcement in the operant chamber with the house light illuminated. If the rat pressed one lever over five times more than the other lever, the animal would not be rewarded, forcing the animal to press the other lever for a reward and preventing the development of a side bias. Once the animals emitted at least 50 responses on both levers for 2 consecutive days, training in the sustained attention task was begun.

\section{Sustained attention task shaping}

Training sessions consisted of a total of 162 trials. Subjects were placed in the operant chambers and given $1 \mathrm{~min}$ to acclimate to the environment. The animals were initially trained to discriminate between signal and nonsignal trials with the house light on throughout the session. Signal and nonsignal trials were presented in a pseudorandomized sequence so that each block of 54 trials consisted of an equal number of signal and nonsignal events. Signal trials consisted of illuminating the central and left panel lights for $1 \mathrm{~s}$. The lights were not illuminated for nonsignal trials. Two seconds after the presentation of the signal or the nonsignal event, both levers were extended into the box and remained for $4 \mathrm{~s}$ or until a lever press occurred. Animals were reinforced for responding to the presence of the light stimuli by depressing the left lever (hit) and the absence of the lights by pressing the right lever (correct rejection). Incorrect lever presses were defined as misses when they occurred on a signal trial and false alarms when they occurred on a nonsignal trial. If the animal failed to respond or responded incorrectly, the levers were retracted and the intertrial interval (ITI) $(12 \pm 3 \mathrm{~s})$ was reinstated. After an incorrect response, the trial was repeated up to three times (correction trials). If the animal failed to respond correctly after three correction trials, a forced-choice trial was initiated. In forced-choice trials, the event (signal or nonsignal) was repeated but only the correct lever was extended and remained active for $90 \mathrm{~s}$. On forced-choice, signal trials, the lights remained illuminated for $90 \mathrm{~s}$. These trials facilitated discriminative conditioning and blocked the development of a side bias. After the animals responded correctly to $\geq 70 \%$ of both the signal and nonsignal events for at least 2 consecutive testing days, they entered a second shaping task. In this task, only the central panel light was illuminated for $1 \mathrm{~s}$.
All other aspects of the task were the same as the previous shaping task. After the animals responded correctly to $\geq 75 \%$ of both the signal and nonsignal events for at least 2 consecutive testing days in this phase of shaping, they entered the final task.

\section{Sustained attention task: baseline task}

In the final version of the task, the length of the signal duration was changed from $1 \mathrm{~s}$ to 25,100 , or $500 \mathrm{~ms}$. Sessions consisted of 27 trials of each of the three signal lengths and 81 trials of the nonsignal trials, yielding a total of 162 trials per session. Because it was planned to analyze performance changes across three blocks of 54 trials each, the sequence of signal and nonsignal trials was pseudorandomized so that one block consisted of 27 signal and 27 nonsignal trials, with each signal length being presented nine times. In addition, both correction and forcedchoice trials were discontinued. Animals were trained to a criterion of $>75 \%$ hits to $500 \mathrm{~ms}$ signals and $>75 \%$ correct rejections to nonsignal trials for at least two consecutive sessions, at which point they were considered ready for surgery.

\section{Surgical procedures}

Rats were paired according to accuracy to nonsignals and the longest signal length. One of each pair was randomly assigned to either receive the immunotoxin 192 IgG-saporin (Advanced Targeting Systems, San Diego, CA) or its vehicle, Dulbecco's saline, by infusion in the prefrontal cortex. Rats were anesthetized using intramuscular injections of ketamine and xylazine ( $85 \mathrm{mg} / \mathrm{kg}$ ketamine and $8.5 \mathrm{mg} / \mathrm{kg}$ xylazine). Infusions of $0.01 \mu \mathrm{g} / \mu \mathrm{l}$ of $192 \mathrm{IgG}$-saporin in Dulbecco's saline or vehicle were made into the prefrontal cortex via a $10 \mu \mathrm{l}, 26$ gauge microsyringe (Hamilton, Reno, NV) mounted on a stereotaxic frame (Stoelting, Wood Dale, IL). A small volume $(0.5 \mu \mathrm{l} / \mathrm{site})$ of the toxin or vehicle was infused bilaterally at a rate of $125 \mathrm{nl} / \mathrm{min}$ using an electronic infusion pump (Micro 4, Microsyringe Pump Controller; World Precision Instruments, Sarasota, FL) to limit any damage of the tissue to that of the toxin. The needle was left in place for $4 \mathrm{~min}$ previous and subsequent to infusion to minimize bleeding and undesired diffusion of the toxin. The following coordinates were used (in $\mathrm{mm}$ ): anteroposterior, bregma +2.8 ; mediolateral, \pm 0.6 ; dorsoventral, skull -5.2 ; and the tooth bar at -3.3 . Animals received $10 \mathrm{~d}$ of ad libitum food and water before food deprivation was reinstated. Four days of additional food deprivation $(\sim 18 \mathrm{~g} / \mathrm{d})$ were established before postoperative training commenced.

\section{Postoperative training}

Animals were retrained to criterion performance on the standard task after recovery from surgery. When rats performed at criterion performance ( $>75 \%$ hits $500 ;>75 \%$ correct rejections) for 2 consecutive days, variations of attentional demands began. After the completion of a testing session, rats were returned to training in the standard task and again required to perform at criterion levels for $2 \mathrm{~d}$ before the next testing session. The order of the six variations of attentional load, as described in detail below, were counterbalanced across subjects to control for possible effects of testing sequence.

\section{Effects of task-irrelevant stimuli}

Previous studies have shown that the effects of flashing house light on attentional performance are so potent that the accuracy of nonlesioned rats in these sessions is similar to the performance of rats after cholinergic depletion of the neocortical mantle during the standard task (McGaughy and Sarter, 1995a; McGaughy et al., 1996). To prevent floor effects on behavior, the modality of the distractor was changed from visual to auditory. The tone was presented at a constant rate $(0.5 \mathrm{~Hz}$; "regular tone") throughout the session or with varying duration and rate of presentation throughout the session $(0.25,0.5,1.5,1,2$, or $3 \mathrm{~s}$ on-off; "irregular tone"). To allow comparison with previous work, we also included tests with flashing house light with one session of a $0.5 \mathrm{~Hz}$ light ("regular light") and one test with a light of varying duration and frequency identical to the irregular tone $(0.25,0.5,1.5,1,2$, or $3 \mathrm{~s}$ on-off; "irregular light").

\section{Effects of dynamic stimulus range}

In the original characterization of the task, a dynamic stimulus range was implemented to heighten attentional load by providing multiple sources 
of targets (McGaughy and Sarter, 1995a,b). A more recent framework describes this variability in targets as expected uncertainty and has hypothesized that acetylcholine is critical to performing under these conditions (Yu and Dayan, 2005). However, confusion exists as to whether it is simply the salience of the $25 \mathrm{~ms}$ signal (Muir et al., 1994) or its position among multiple targets that taxes attention. To assess the effects of expected uncertainty versus simple signal salience on performance, the dynamic stimulus range was removed so that sessions consisted of only $25 \mathrm{~ms}$ signals and nonsignals with the expectation that the animals would be better at detecting the $25 \mathrm{~ms}$ signal when it was not embedded in the dynamic stimulus range.

\section{Effects of an increase in the variability of the ITI}

Event asynchrony degrades performance efficiency when compared with regular event schedules (Scerbo et al., 1987; McGaughy and Sarter, 1995a). Because the variability of the ITI was kept relatively small (12 \pm $3 \mathrm{~s}$ ), it could not be completely excluded that the animals were able to approximate the onset of the signal or nonsignal event. Therefore, the effects of an increase in the variability of the ITI to $12 \pm 6 \mathrm{~s}$ were examined. Animals were tested for one session in the task using the highly variable ITI. This session allowed the assessment of increased attentional demands different from those of the task-irrelevant stimuli.

\section{Characterization of the effects of tone on performance}

After all of the above variations were completed, the consistent tone was found to improve attentional performance. We hypothesized that rats used the regularity of the $0.5 \mathrm{~Hz}$ tone to time when to attend. To test this hypothesis, the consistent $0.5 \mathrm{~Hz}$ tone was presented in combination with the highly asynchronous event rate. This variation was tested twice. Rats were trained in the standard task after the first session until they reestablished criterion performance.

Data analyses from these previous variations also showed a detrimental effect of the irregular tone on task performance. It was hypothesized that this impairment may result from the coincident presentation of the distractor and target. This hypothesis was tested by assessing the effects of a brief tone $(15 \mathrm{~ms})$ consistently paired with the presentation of the signal or nonsignal event. The order of testing of these two final tests was counterbalanced across subjects, with half of the rats tested first with the tone and highly asynchronous ITI and half with the tone coincident with events.

\section{Behavioral measures}

From each test session, the number of hits, misses, correct rejections, false alarms, and errors of omissions were recorded. The relative number of hits (hits/hits + misses) was calculated for each signal length. Furthermore, the relative number of correct rejections (correct rejections/correct rejections + false alarms) was computed. The relative number of left lever presses (hits + false alarms/all responses) was also calculated to detect any side biases the animal may have developed in which the initial analyses of hits and correct rejections suggested such a bias could explain the pattern of results.

Histology. After completion of testing, animals were given an overdose of ketamine and xylazine ( $100 \mathrm{mg} / \mathrm{kg}$ ketamine and $10 \mathrm{mg} / \mathrm{kg}$ xylazine). The rats were then transcardially perfused with $0.9 \%$ saline, followed by $4 \%$ paraformaldehyde in phosphate buffer. Brains were removed and placed in a 30\% sucrose solution until they sunk and then sliced into 50 $\mu \mathrm{m}$ coronal sections. Alternate sections were thionin stained for Nissl bodies and acetylcholinesterase (AChE) as optimized for cortical fibers as described previously (Tago et al., 1986). Briefly, sections were placed in phosphate buffer, $\mathrm{pH} 7.4$, with $0.1 \% \mathrm{H}_{2} \mathrm{O}_{2}$ before rinsing with $0.1 \mathrm{M}$ maleate buffer (three times for $3 \mathrm{~min}, \mathrm{pH}$ 6.0). Sections were then incubated in a solution of $5 \mathrm{mg}$ of acetylthiocholine idodide, $0.147 \mathrm{~g}$ of sodium citrate, $0.075 \mathrm{~g}$ of copper sulfate, and $0.0164 \mathrm{~g}$ of potassium ferricyanide in 0.1 maleate buffer for $60 \mathrm{~min}$. After incubation, sections were rinsed in $50 \mathrm{~mm}$ Tris buffer (three times for $3 \mathrm{~min}, \mathrm{pH}$ 7.6). A second incubation then commenced using $0.05 \mathrm{~g}$ of diaminobenzidine and 0.375 $\mathrm{g}$ of nickel ammonium sulfate in $125 \mathrm{ml}$ of $50 \mathrm{~mm}$ Tris buffer. Twelve drops of $0.1 \% \mathrm{H}_{2} \mathrm{O}_{2}$ were added to each $60 \mathrm{ml}$ bath until cortical layers became visible. Sections were rinsed in $5 \mathrm{~mm}$ Tris buffer (three times for $3 \mathrm{~min}$ ) before mounting with gelatin on gelatin-coated slides. Histolog- ical assessment of fiber loss was made using a modified grid counting procedure (McGaughy et al., 1996; Ross et al., 2005). Sections were examined on an Olympus Optical BX51 microscope (Optical Analysis Corporation) using the $40 \times$ objective. Images of the sections were photographed using a SPOT Insight digital camera (Diagnostic Imaging, Sterling Heights, MI). A grid measuring $300 \times 300 \mu \mathrm{m}$ was applied to the image of the brain using Image Pro Plus version 6.0 software (Media Cybernetics, Silver Springs, MD), and the number of fibers that crossed the perimeter of the grid were counted (McGaughy et al., 2005; Ross et al., 2005). Fiber counts were obtained in the infralimbic/prelimbic cortices (IL/PL) at bregma $+4.7,+3.7,+2.7$, and $+2.5 \mathrm{~mm}$ and in the cingulate cortex at bregma $+3.7,+2.5$, and $+0.7 \mathrm{~mm}$.

\section{Statistical analyses}

Histology. All statistical analyses were done on SPSS 14.0 for Windows. The extent of the lesion was determined with a separate ANOVA for each region (IL/PL and cingulate). The extent of damage in a region was assessed in a mixed-factors ANOVA with hemisphere (2 levels) and rostrocaudal position (IL/PL, 4; cingulate, 3 ) as within-subject factors and lesion (2) as the between-subjects factor.

\section{Behavioral analyses}

Postsurgical baseline sessions. All dependent measures were analyzed using separate mixed-factor ANOVAs. The degrees of freedom in all analyses were corrected using the Huynh-Feldt correction in the case of a violation of sphericity. $\varepsilon$ values not equal to 1 are reported below. The mean performance on the baseline days before each attentional variation day were compared with each other to determine whether there was any difference in the performance of ACh-LX and Sham-LX rats in postsurgical performance in the standard task over time. For the analysis of the effects of time on task (vigilance decrement), test sessions were divided in three blocks of 54 trials (see above). The effects of signal length and block over the days of baseline on hit accuracy were analyzed using a mixedfactor ANOVA with one between-subjects factor (lesion, 2) and three within-subject factors (day, 9; block, 3; signal, 3). Note that the independent variable day has nine levels because it includes the mean performance for the $2 \mathrm{~d}$ before each behavioral variation in addition to a second baseline day from the test of a $0.5 \mathrm{~Hz}$ tone and highly asynchronous event rate as rats were exposed to this test twice. The effects of block on correct rejection accuracy were analyzed using a mixed-factors ANOVA with one between-subjects factor (lesion, 2) and two within-subject factors (day, 9; block, 3).

Sessions with varied attentional demand. Baseline performance for each dependent measure was calculated using mean of the two sessions in the standard task (ITI, $12 \pm 3 \mathrm{~s}$ ) immediately before a test of altered attentional demand (e.g., regular tone) so that each ANOVA has two levels, allowing a comparison of the mean baseline performance to the test session. The effects of varied attentional demand were assessed in independent, mixed-factors ANOVAs with one between-subjects factor (lesion, 2) and three within-subject factors (task variation, 2; block, 3; signal, 3). The effects on correct rejection accuracy were analyzed using a mixed-factors ANOVA with one between-subjects factor (lesion, 2) and two within-subject factors (task variation, 2; block, 3).

Event asynchrony with $0.5 \mathrm{~Hz}$ tone. The effects of increasing the event asynchrony from $12 \pm 3$ to $12 \pm 6$ s ITIs in the presence of a consistent tone $(0.5 \mathrm{~Hz})$ was tested for two sessions. Means for each dependent variable for the $2 \mathrm{~d}$ before the first test session were calculated so that the factor high asynchrony tone had three rather than two levels. A mixedfactors ANOVA was used to analyze the effects of this variation on performance with three within-subjects factors high asynchrony tone (3), block (3), and signal (3) and one between-subjects factor lesion (2). The effects on correct rejection accuracy were analyzed using a mixed-factor ANOVA with one between-subjects factor (lesion, 2) and two withinsubject factors (high asynchrony tone, 3 ; block, 3 ).

\section{Results \\ Histology}

The lesions effectively decreased the number of $\mathrm{AChE}^{+}$fibers in the IL/PL but not the cingulate region of the frontal cortex (le- 


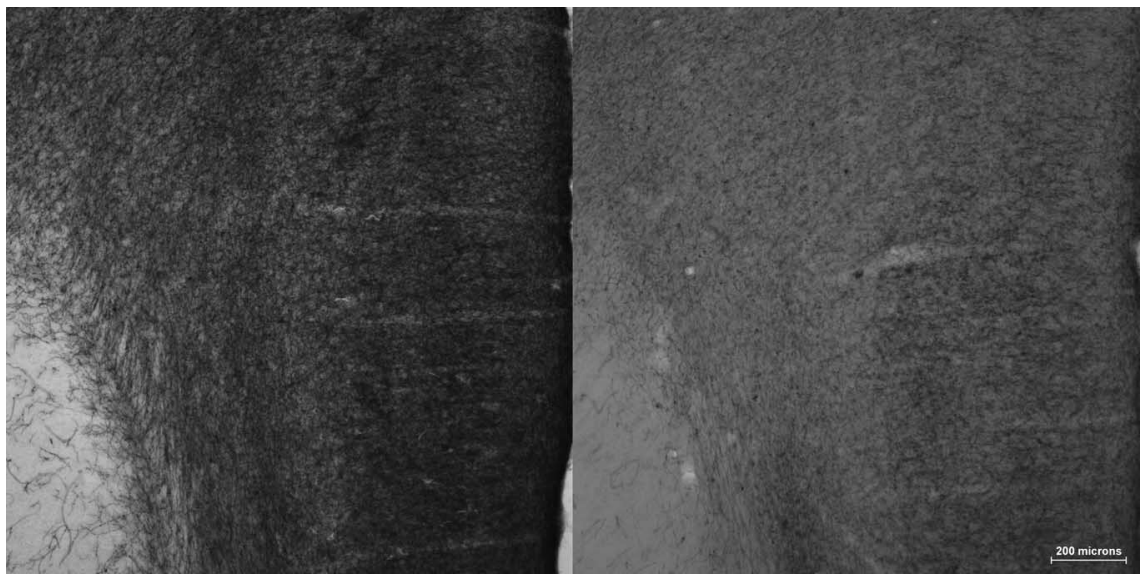

Figure 1. Cortical cholinergic fibers as elucidated by AChE staining from the IL/PL. Although all rats received bilateral infusions of either $192 \mathrm{lgG}$-saporin or its vehicle, for illustration purposes, the left side of the figure shows fibers from a Sham-LX rat, and the right side shows fibers from an ACh-LX rat. Fiber loss was consistent in the IL/PL in ACh-LX rats rostral to caudally with an average loss of $48 \%$ in cortical fibers as assessed using a modified grid counting technique. Loss of AChE-positive fibers was restricted to the region of the IL/PL, and the toxin did not damage the cingulate cortex dorsal to this region. Fiber counts were taken slightly lateral to the midline to allow the assessment of loss in the IL/PL in regions not innervated by the brainstem cholinergic nuclei, which was spared in the current study.

\section{Baseline}

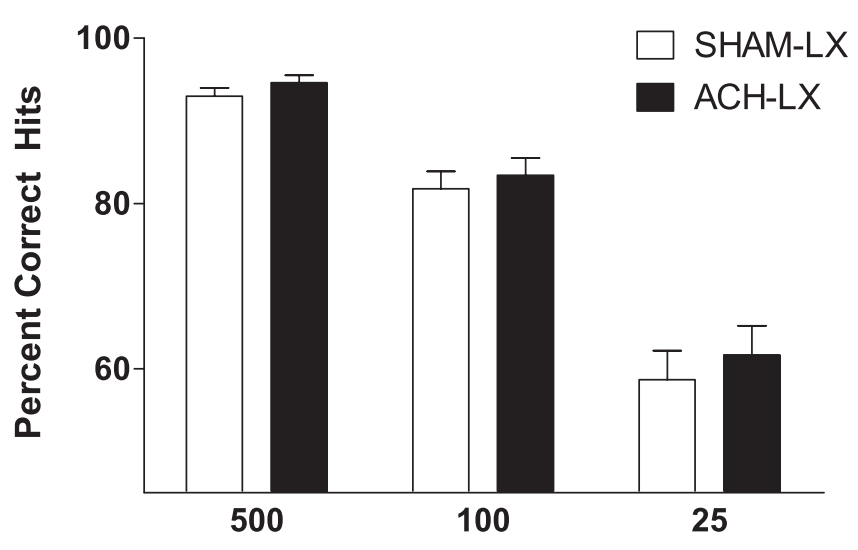

\section{Signal Length}

Figure 2. During the baseline testing sessions, there was no difference between the performance of Sham-LX (white bars) and ACh-LX (black bars) on signal trials regardless of signal length $(500,100$, or 25 ms signals).

sion: IL/PL, $F_{(1,14)}=547.02, p<0.001$; cingulate cortex $1, F_{(1,14)}$ $=0.51, p=0.48)$. The ACh-LX rats had an average loss of $48.5 \%$ in the region of the IL/PL relative to Sham-LX rats (mean \pm SEM: ACh-LX, $58.98 \pm$ 1.64; Sham-LX, $114.45 \pm 1.72$ ) (Fig. 1). The loss of fibers did not vary based on hemisphere (all $p>0.10$ ) or rostrally to caudally (all $p>0.08$ ).

\section{Baseline}

Cholinergic deafferentation of the prefrontal cortex did not produce any differences in responses to signals (lesion, $F_{(1,14)}=0.54$; $p=0.48$ ) (Fig. 2) or nonsignals (lesion, $F_{(1,14)}=0.002 ; p=0.96$ ). The effects of lesion were unchanged regardless of signal length, the amount of time on task, or the day of testing (all $p>0.10$ ). Performance was found to be signal length dependent as shown previously $\left(F_{(2,28)}=207.69 ; p<0.001 ; \varepsilon=0.73\right)$ (Fig. 2).

\section{Task-irrelevant tones}

There was a differential sensitivity of the ACh-LX rats to irregular tone relative to Sham-LX rats over the course of the testing session $\left(F_{(2,28)}=5.74 ; p=0.03\right)$. The performance of ACh-LX rats in the presence of the distractor during the initial block of trials was significantly impaired relative to performance in the same block in the standard task $\left(t_{(7)}=3.99 ; p=0.005\right.$; corrected $\alpha=0.017$ ) (Fig. 3). The Sham-LX rats' performance was unaffected by the presence of the irregular tone over the course of the testing session (all $p>0.57$ ) (Fig. 3). The effects of the variable tone produced no differential effects on the performance of Sham-LX and ACh-LX rats in the detection of signals (all $p>0.06$ ). The presence of the irregular tone produced no effects on response to nonsignals (all $p>0.16$ ).

The regular $(0.5 \mathrm{~Hz})$ tone facilitated detection of signals $\left(F_{(1,14)}=7.29 ; p=0.02\right)$, and this beneficial effect was greater with prolonged time on task $\left(F_{(2,28)}=5.33 ; p=\right.$ $0.01 ; \varepsilon=0.89$ ) (Fig. 4). Planned comparisons of performance in the presence of the regular tone during the third block of trials was greater than under baseline conditions $\left(t_{(15)}=4.49 ; p<\right.$ 0.001 ; corrected $\alpha=0.017)$. This improvement in performance in the third block differed between the groups $\left(F_{(2,28)}=4.53 ; p=\right.$ 0.02 ). Additionally, Sham-LX and ACh-LX showed improvements at different signal lengths during the third block of trials (lesion $\times$ signal length $\times$ block, $F_{(4,56)}=2.68 ; p=0.04$ ). Analyses of performance in the third block of trials in the presence of the regular tone revealed that Sham-LX rats detected the $25 \mathrm{~ms}$ signal with greater accuracy $\left(t_{(7)}=-3.38 ; p=0.012\right.$; corrected $\alpha=0.017$ ) (Fig. 5), whereas ACh-LX rats showed an improved detection of the $100 \mathrm{~ms}$ signal $\left(t_{(7)}=-3.33 ; p=0.013\right.$; corrected $\alpha=0.017$ ) as shown in Figure 6. There was no effect of the $0.5 \mathrm{~Hz}$ tone on responses to nonsignals $\left(F_{(1,14)}=2.27 ; p=0.15\right)$, and there were no other significant main effects or interactions in this analysis (all $p>0.21)$.

\section{Task-irrelevant lights}

There was no differential response of ACh-LX rats to the effects of flashing house light when compared with Sham-LX rats. As found in previous studies, flashing house light significantly impaired the detection of signals regardless of whether the light flashed in a regular $\left(F_{(1,14)}=66.79 ; p<0.001\right.$; mean \pm SEM: baseline, $76.0 \pm 2.0$; regular light, $45 \pm 4.0$ ) or irregular pattern $\left(F_{(1,14)}=35.59 ; p<0.001\right.$; baseline, $87.0 \pm 2.0$; irregular light, $62.7 \pm 4.0)$. The detrimental effects of the task-irrelevant lights did not interact with the effects of lesion (all $p>0.27$ ) or with the effects of time on task (all $p>0.16$ ). Correct rejections were also significantly decreased in the presence of the $0.5 \mathrm{~Hz}$ light $\left(F_{(1,14)}\right.$ $=9.14 ; p<0.001$; baseline, $87.0 \pm 0.8$; regular light, 78.0 \pm 3.0$)$ and the irregular, flashing house light $\left(F_{(1,14)}=9.14 ; p=0.009\right.$; baseline, $87.0 \pm 2.0$; irregular light, $62.0 \pm 4.0)$. This effect did not differ between groups (all $p>0.17$ ).

\section{Removal of the dynamic stimulus range}

The presentation of the $25 \mathrm{~ms}$ signal alone facilitated detection of this stimulus $\left(F_{(1,14)}=10.28 ; p=0.006\right.$; mean \pm SEM: baseline $25 \mathrm{~ms}, 59.0 \pm 4.0$; only $25 \mathrm{~ms}, 69.0 \pm 2.0$ ). This effect was un- 
SHAM-LX RATS

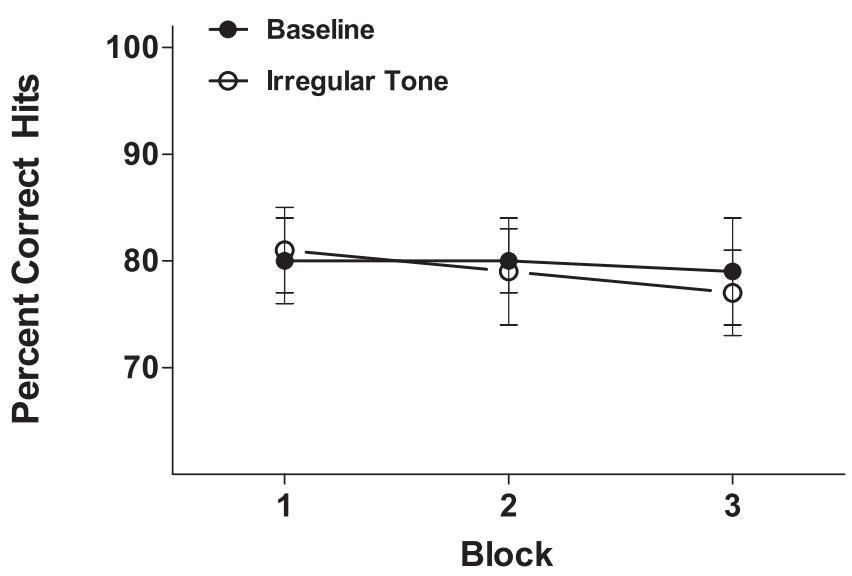

ACH LX

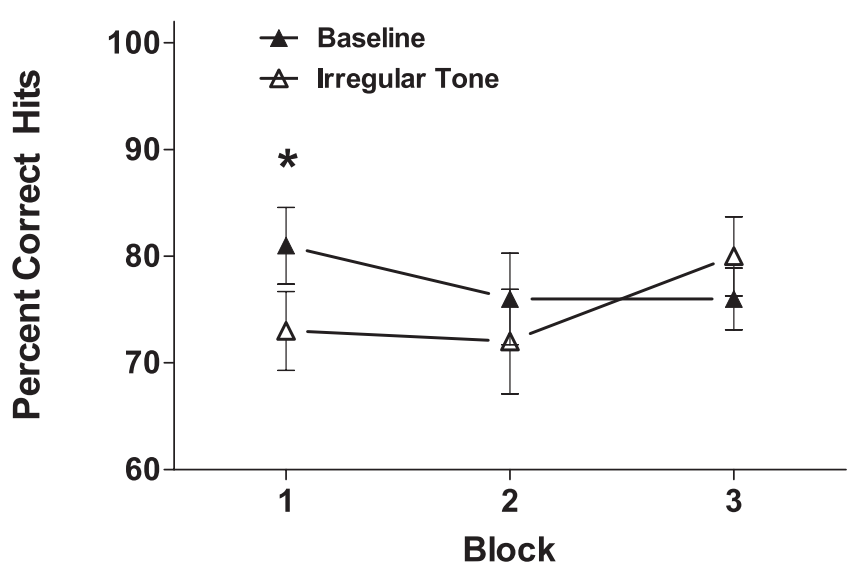

Figure 3. An irregularly occurring tone disrupted performance of $A C h-L X$ rats (right, open symbols) in the first but not subsequent blocks of trials. This task-irrelevant stimulus had no effect on the performance of Sham- $\mathrm{LX}$ rats (left). The transience of the impairment in ACh-LX rats suggest that top-down control of attention that allows subject to disregard task-irrelevant stimuli was compromised but not abolished. ${ }^{*} p<0.05$.

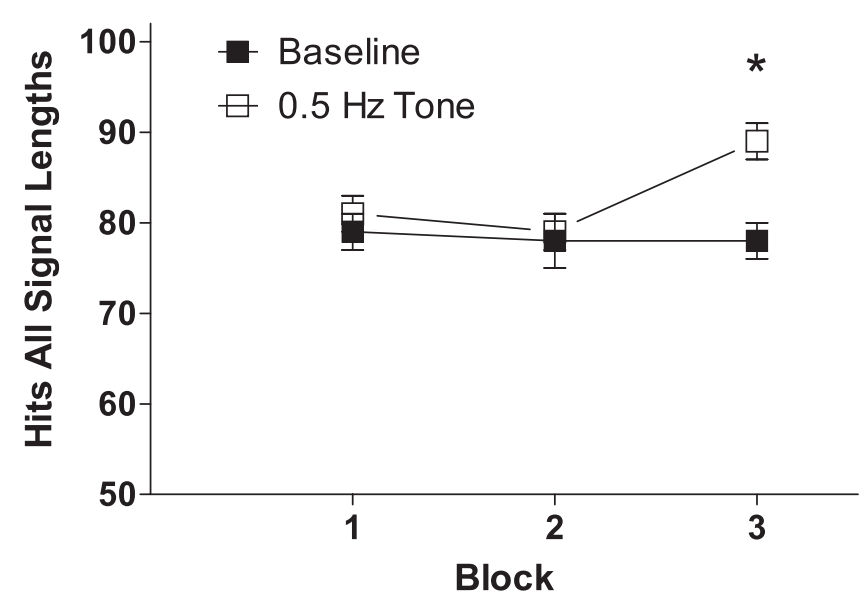

Figure 4. A $0.5 \mathrm{~Hz}$ tone significantly improved detection of signals in the third block for both groups of rats (open diamonds) relative to baseline conditions (filled diamonds). Although both groups showed improvements in performance in block 3 , this benefit was seen at different signal lengths, as shown in Figures 4 and 5.
SHAM LX RATS 25 MSEC

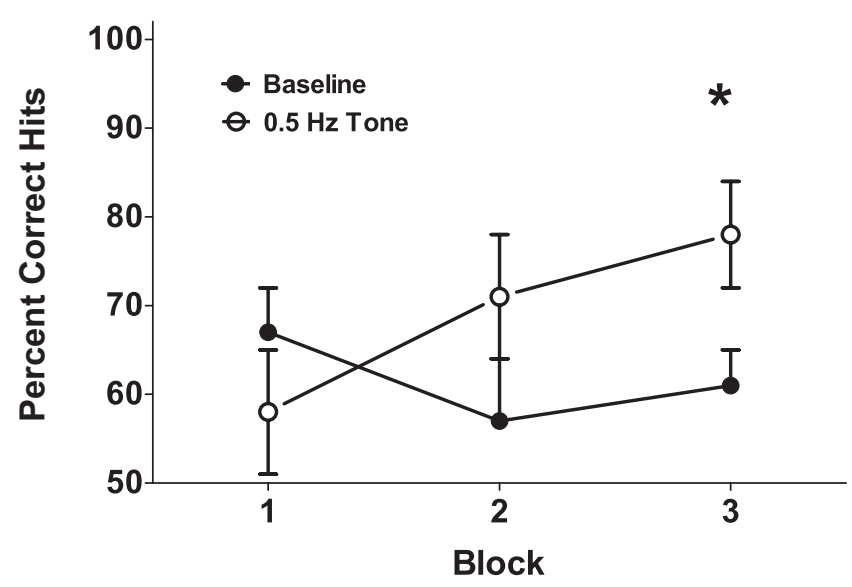

ACH LX 25 MSEC

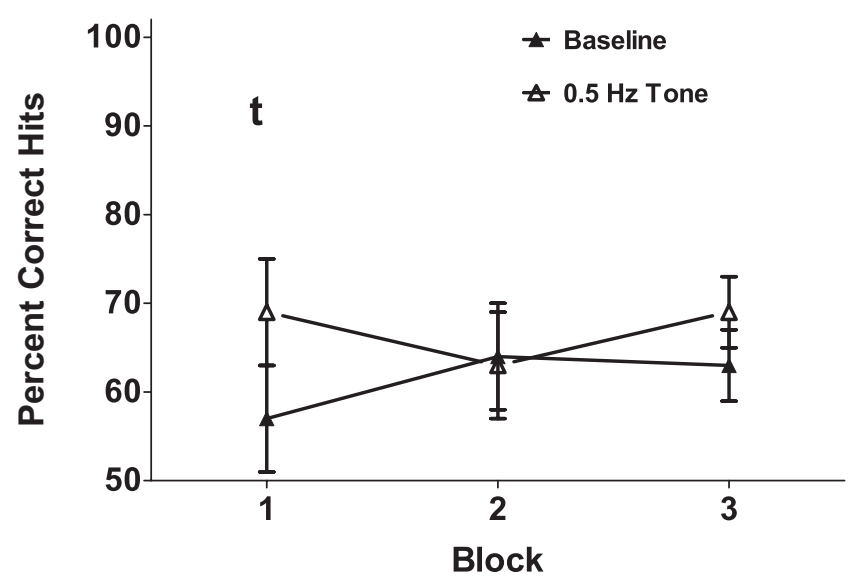

Figure 5. Detection of the $25 \mathrm{~ms}$ signal during baseline (open symbols) and sessions with the $0.5 \mathrm{~Hz}$ tone (filled symbols) for Sham- LX (left) and ACh- LX (right) rats over the course of the testing session. The introduction of a $0.5 \mathrm{~Hz}$ tone facilitated Sham- $L X$ but not ACh-LX rats' detection of the $25 \mathrm{~ms}$ stimulus during block 3 . There was a trend for ACh-LX rats' detection of the 25 ms signal to be improved in block $1 .{ }^{*} p<0.05$.

changed by the lesion (all $p>0.37$ ) (Fig. 7) or time on task (all $p>0.73$ ). There was a statistically significant although small impairment in the response to nonsignal during the session with only 25 ms signals $\left(F_{(1,14)}=6.07 ; p=0.03\right.$; mean \pm SEM: baseline, $88.0 \pm 1.0$; only $25 \mathrm{~ms}, 85.0 \pm 1.0$ ). This effect did not differ between lesion groups (all $p>0.17$ ) or change with increased time on task (all $p>0.16$ ).

\section{High asynchrony}

The effects of high asynchrony failed to interact with lesion $\left(F_{(1,14)}=0.19 ; p=0.67\right)$, but the effects of high asynchrony over time on task differed between the Sham-LX and ACh-LX rats $\left(F_{(2,28)}=5.80 ; p<0.008\right)$. This effect did not result from impairment in performance under conditions of high asynchrony in ACh-LX rats (all $p>0.15$ ); rather, there was a transient improvement in the detection of signals in Sham-LX rats during block 2 of the more variable ITI condition relative to baseline $\left(t_{(7)}=3.14\right.$; $p>0.016$; correct $\alpha=0.017)$. There were no other significant effects or interactions in the analysis of hits (all $p>0.08$ ). The response to nonsignals was unchanged by this variation regardless of lesion group (all $p>0.09$ ). 

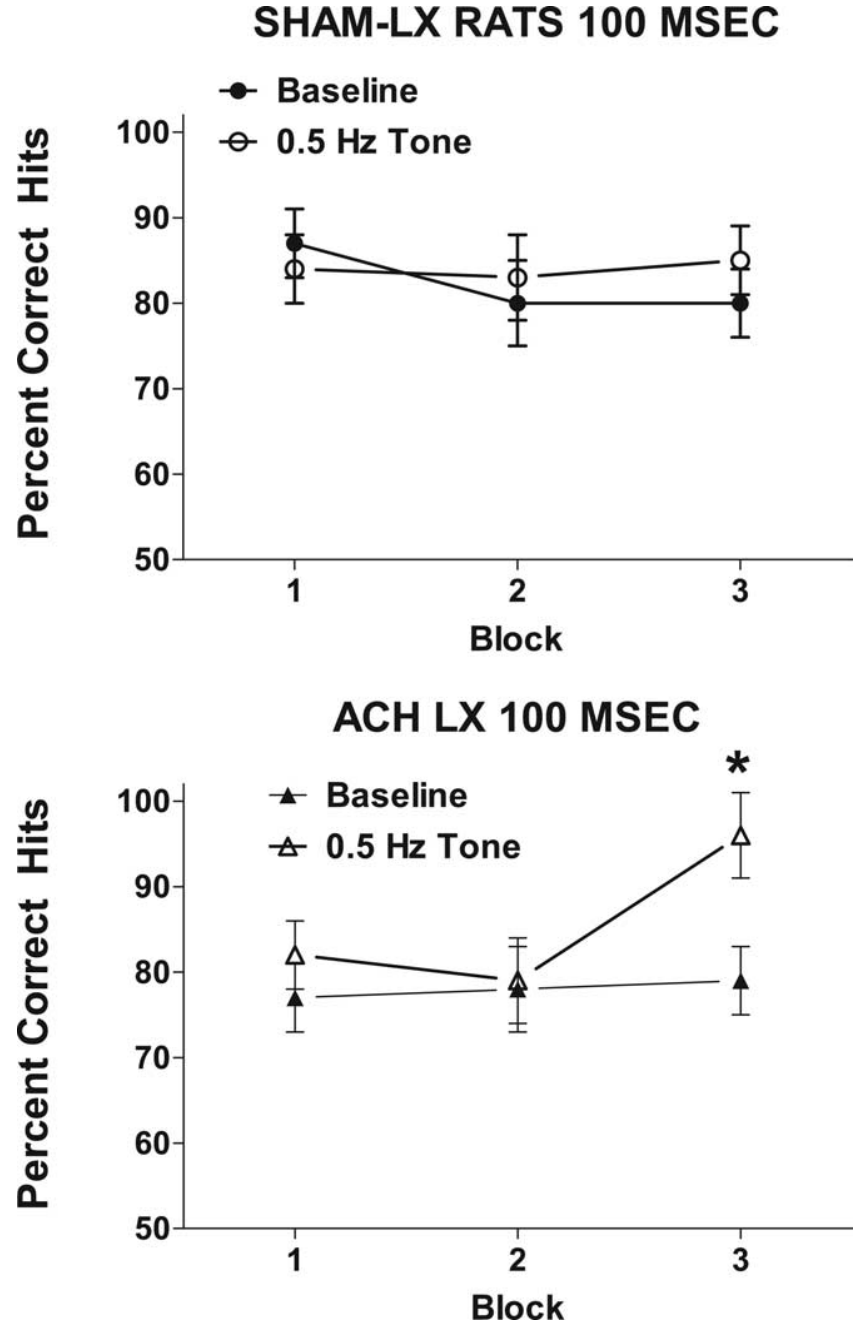

Figure 6. Detection of the $100 \mathrm{~ms}$ signal during baseline (open symbols) and sessions with the $0.5 \mathrm{~Hz}$ tone (filled symbols) for Sham-LX (left) and ACh-LX (right) rats over the course of the testing session. The introduction of a $0.5 \mathrm{~Hz}$ tone facilitated ACh-LX rats' detection of the $100 \mathrm{~ms}$ stimulus, whereas Sham- $\mathrm{LX}$ rats detection of the $100 \mathrm{~ms}$ signal was unchanged by the $0.5 \mathrm{~Hz}$ tone. ${ }^{*} p<0.05$.

High asynchrony with regular tone

The initial day of high asynchrony in the presence of the regular tone impaired performance $\left(F_{(2,28)}=8.59 ; p=0.001 ; \varepsilon=0.92\right.$; mean \pm SEM: baseline, $80.5 \pm 2.0$; high asynchrony/tone day 1 , $66.9 \pm 9.0$; high asynchrony/tone day 2, 79.2 \pm 3.0 ). This impairment on day 1 of the highly asynchronous ITI with tone was greater in ACh-LX rats relative to Sham-LX $\left(F_{(2,28)}=4.03 ; p=\right.$ $0.03 ; \varepsilon=0.92$; baseline vs high asynchrony/tone day 1 : Sham-LX, $t_{(7)}=1.35 ; p=0.22$; ACh-LX, $t_{(7)}=2.72 ; p=0.03$ ) (Fig. 8). The effects of the combination high asynchrony and regular tone did not interact with any other factor in the analyses of hits (all $p>$ 0.64 ) and produced no effects on performance of nonsignals (all $p>0.31)$.

Brief tone coincident with events

The presentation of a briefly overlapping tone with signals did not produce any change in hits in either ACh-LX or Sham-LX rats (all $p>0.08$ ). The presentation of a brief tone coincident with nonsignals produced similar effects on correct rejections in both Sham-LX and ACh-LX (all other $p>0.29$ ).

\section{MSEC STIMULI}

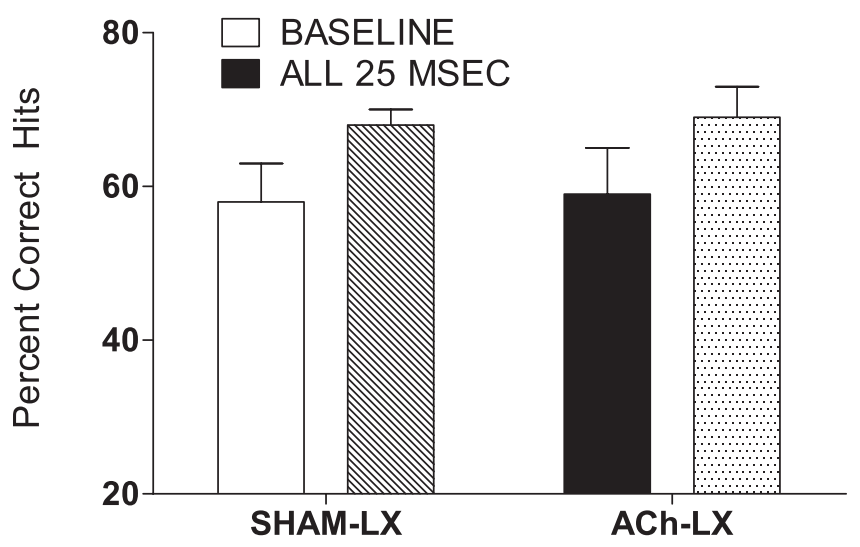

Figure 7. The abolition of the dynamic stimulus range improved detection of the $25 \mathrm{~ms}$ signal. Performance in the standard task for Sham-LX (white bar) and ACh-LX (black bar) rats is similar, as is the improvement in performance in the session in which only the $25 \mathrm{~ms}$ signal is presented (lined bars). These data support the hypothesis that it is the dynamic stimulus range, not the sensory attributes of the $25 \mathrm{~ms}$ signal, that make it most difficult to detect in the baseline sessions of the task.

0.5 Hz Tone with High Asynchrony ITI

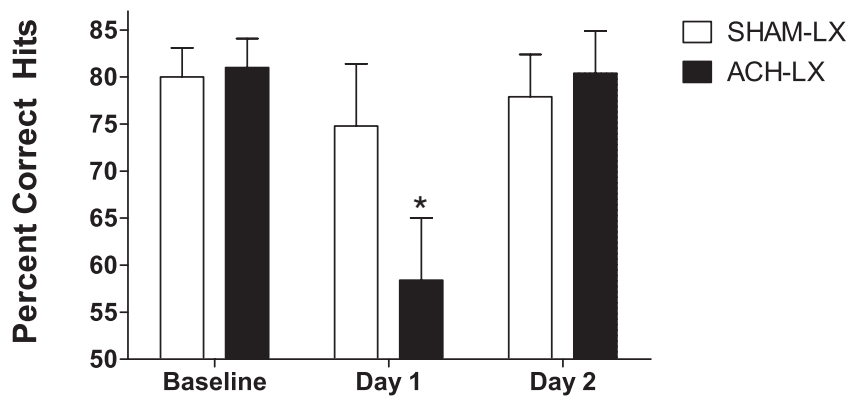

Figure 8. The beneficial effect of the $0.5 \mathrm{~Hz}$ tone (shown in Fig. 3) was disrupted in the first session; it was paired with an increase in event asynchrony in both Sham-LX (white bars) and $A C h-L X$ (black bars). This suggests that the regularity of the tone allowed subjects to time when to attend. This impairment was greater in ACh-LX rats than Sham-LX rats and is hypothesized to reflect compromised top-down control in these subjects. Both groups soon adapted their behavior so that this pairing of the $0.5 \mathrm{~Hz}$ tone with the highly asynchronous event rate no longer impaired performance on the second exposure to these conditions (Day 2), but the increase in temporal unpredictably prevented the beneficial effects of the tone. ${ }^{*} p<0.05$.

\section{Discussion}

Restricted cholinergic lesions of IL/PL, without concurrent damage to cingulate cortex, increase susceptibility to distraction in the absence of baseline attentional impairments (Fig. 2). Intra-nbm infusions of 192 IgG-saporin produced robust impairments in detection of all signals (McGaughy et al., 1996, 1999; McGaughy and Sarter, 1999), whereas diffuse intracortical infusions of the cholinotoxin impaired detection of $500 \mathrm{~ms}$ signals (McGaughy and Sarter, 1998). Our data show that IL/PL damage alone is sufficient to impair attention, suggesting that it has a unique role in attention. Previous work has shown that cholinergic deafferentation of IL/PL, but not orbitofrontal, impedes formation of an attentional set (McGaughy et al., 2002b). We restricted the size of lesions in the present study to preserve baseline attentional performance and confine damage to IL/PL. Similar cholinergic lesions of IL/PL and cingulate cortices impair attention for action (Maddux et al., 2007). Differences in task requirements, the larger amount of cholinergic deafferentation, or concurrent 
damage to cingulate cortex may contribute to the larger attentional impairments attention for action (Chudasama et al., 2003; Maddux et al., 2007).

A $0.5 \mathrm{~Hz}$ tone improved signal detection in all rats when attentional demands were high, e.g., late in the testing session with shorter duration targets (Figs. 4-6). Additional tests suggest that rats use the tone to time when to attend. This benefit is hypothesized to depend on an intact PPC because it has been shown to control timing of attention (Coull and Nobre, 1998). Increased event asynchrony alone spared attentional performance, but the first exposure to increased event asynchrony with the $0.5 \mathrm{~Hz}$ tone impaired performance. ACh-LX rats showed a greater deficit than Sham-LX rats under these conditions, suggesting that they were more reliant on the tone (Fig. 8). An irregular tone is detrimental to the performance of ACh-LX rats but not Sham-LX rats. This impairment is transient, and ACh-LX rats disregard the irregular tone after one block of trials (Fig. 3). These data support the hypothesis that cholinergic lesions of PFC increase susceptibility to cross-modal distraction.

Visual distractors potently impaired performance of all rats. The perceptual similarity between visual distractors and targets likely augments their disruptive effects. The irregular light has flashes of house light illumination identical to the duration of targets. False alarms were higher in these sessions (37\%) than in sessions with a regularly flashing light (22\%), suggesting that increased perceptual similarity yields greater confusion between targets and distractors. Both the tone generator and house light are on the wall opposite the panel lights, but the reflection of the house light throughout the box makes it more ubiquitous and difficult to ignore than the tone. We hypothesize that the differences in performance between visual and auditory stimuli were related to issues of salience and that all performance with distractors requires the same attentional network (Shomstein and Yantis, 2004, 2006; Buschman and Miller, 2007). Maintenance of attention in the presence of distraction requires a network of activation including both PFC and PPC (Coull, 1998; Coull and Nobre, 1998; Mesulam, 1999; Bunge et al., 2002; Assad, 2003; Chambers et al., 2004; Buschman and Miller, 2007), and cholinergic lesions of the PFC disrupt this network.

The increased sensitivity of ACh-LX rats to task-irrelevant stimuli could originate from two possible effects of cholinergic deafferentation in the PFC. First, cholinergic deafferentation of PFC may decrease top-down attentional control and prohibit filtering of irrelevant stimuli. Specifically, damage to the PFC disrupts descending control of the cholinergic basal forebrain to prevent augmented cholinergic efflux in PPC that aids in resolving targets from distractors (Sarter et al., 2001a, 2005; Nelson et al., 2005; Buschman and Miller, 2007). Alternatively, decreased acetylcholine in PFC produces a shift in circuit dynamics to ultimately increase the influence of corticocortical circuits, specifically PPC to PFC, that mediate bottom-up processing of stimuli (Hasselmo and McGaughy, 2004; Buschman and Miller, 2007). Subsequently, we discuss the hypothesized effects of decreased top-down processing and increased bottom-up processing in turn.

If cholinergic deafferentation abolished top-down control of attention, all task-irrelevant stimuli would impair performance because targets could not be discriminated from distractors and performance would remain unchanged despite training. The present data suggest that cholinergic deafferentation of PFC compromises, but does not abolish, top-down control of attention. Evidence of residual top-down control in ACh-LX rats includes their ability to disregard the irregular tone after one block of trials and to disregard unimodal distractors to the same degree as Sham-LX rats. Support for the hypothesis that cholinergic lesions of IL/PL compromise top-down control comes from the initial test of the $0.5 \mathrm{~Hz}$ tone with the highly asynchronous ITI. ACh-LX rats, but not Sham-LX rats, have impairments in this session. The diminished ability of ACh-LX rats to adjust to the change in the relationship between the tone and event timing suggests compromised top-down control. Top-down processing of stimuli in the presence of increased attentional demands is hypothesized to result from high levels of prefrontal acetylcholine activating descending glutamatergic pathways from the cortex to the nbm and ultimately augmenting cholinergic efflux in PPC (Nelson et al., 2005). The loss of PFC acetylcholine in the present study would prevent increased cholinergic efflux in PPC (Sarter and Bruno, 1997, 1999; Sarter et al., 1999, 2001b, 2005), impeding discrimination of targets from distractors. However, a loss of top-down control cannot account for the differential effects of distractors based on the regularity and modality of these stimuli.

We hypothesize that these effects reflect enhanced bottom-up processing of stimuli. Prefrontal ACh biases responding to behaviorally relevant stimuli relayed via subcortical afferents rather than corticocortical afferents (Hasselmo and Bower, 1992; Hasselmo, 1999; Hasselmo and McGaughy, 2004). Highly salient stimuli result in attentional "capture" and produce neural activation in PPC before activation in PFC (Buschman and Miller, 2007). We hypothesize that this sequence of firing suggests that information about novel, salient distractors is relayed from PPC to PFC in all rats. Cholinergic deafferentation of PFC increases the influence of this circuit on behavior and underlies the increased susceptibility of ACh-LX rats to cross-modal distraction. In contrast to Sham-LX rats, ACh-LX rats show beneficial effects of the $0.5 \mathrm{~Hz}$ tone in the first block of trials. The immediacy with which this tone improves performance in ACh-LX rats cannot be explained by compromised top-down, attentional processing. Additionally, the differential effects of regular and irregular tones in ACh-LX rats show that the perceptual properties of stimuli influence its effects. These data suggest that bottom-up processing in ACH-LX rats is increased. We hypothesize that this greater bottom-up processing of task-irrelevant stimuli in lesioned rats reflects a larger influence of PPC on behavior attributable to a shift in circuit dynamics after cortical, cholinergic deafferentation of PFC.

Other studies have shown that PPC neurons respond to target, but not task-irrelevant, stimuli (Broussard et al., 2006), whereas PFC neurons respond to target and distractor stimuli (Gill et al., 2000). A critical methodological difference may account for the discrepancy between previous work and our finding. In the previous study, recordings were made after the rats were repeatedly exposed to the distractor. Repeated exposure to a distractor produces performance of $\sim 70 \%$ accuracy in detecting signals in the presence a $0.5 \mathrm{~Hz}$ flashing house light (Gill et al., 2000; Broussard et al., 2006). In contrast, our Sham-LX subjects accurately detected $40 \%$ of signals during the flashing house-light session. Because baseline performance of rats is similar across studies, it seems that the difference in the flashing house-light session is attributable to repeated training with this stimulus. We hypothesize that PPC neurons would increase firing before PFC neurons during the initial exposure to a highly salient distractor of any modality (Buschman and Miller, 2007). When animals learned to distinguish behaviorally relevant from irrelevant stimuli, increased firing would be found in PFC before PPC neurons (Buschman and Miller, 2007). Our data suggest that bottom-up processing of these visual distractors is so potent that even rats 
with fully intact prefrontal cortex cannot maintain attentional performance. Repeated exposure to these visual distractors may dissociate the performance of ACh-LX and Sham-LX rats. Future studies may be aimed at determining whether lesions of the PPC prevent the attentional effects of all task-irrelevant stimuli and whether PPC and PFC firing to these distractors changes after repeated testing.

We also assessed the effects of cholinergic deafferentation of IL/PL on expected uncertainty. Cortical acetylcholine is hypothesized to mediate responses to expected uncertainty, but we found no difference between groups when expected uncertainty was reduced (Yu and Dayan, 2002, 2005). Detection of the shortest signal was improved when it was presented alone for all rats (Fig. 7). This finding suggests that the dynamic stimulus range increases attentional demand and compromises detection of less salient stimuli. It is unclear whether increases in expected uncertainty would impair performance after cholinergic, prefrontal deafferentation, but the addition of more targets in future studies may address this question. In summary, cholinergic lesions of IL/PL decrease top-down and increase bottom-up attentional control, but changes in expected uncertainty were without effect in ACh-LX rats.

\section{References}

Assad JA (2003) Neural coding of behavioral relevance in parietal cortex. Curr Opin Neurobiol 13:194-197.

Baxter M, Chiba A (1999) Cognitive functions of the basal forebrain. Curr Opin Neurobiol 9:178-183.

Bigl V, Woolf N, Butcher LL (1982) Cholinergic projections from the basal forebrain to frontal, parietal, temporal, occipital, and cingulate cortices: a combined fluorescent tracer and acetylcholinesterase analysis. Brain Res Bull 8:727-749.

Broussard J, Sarter M, Givens B (2006) Neuronal correlates of signal detection in the posterior parietal cortex of rats performing a sustained attention task. Neuroscience 143:407-417.

Bunge SA, Hazeltine E, Scanlon MD, Rosen AC, Gabrieli JDE (2002) Dissociable contributions of prefrontal and parietal cortices to response selection. NeuroImage 17:1562-1571.

Buschman TJ, Miller EK (2007) Top-down versus bottom-up control of attention in the prefrontal and posterior parietal cortices. Science 315:1860-1862.

Chambers CD, Stokes MG, Mattingly JB (2004) Modality-specific control of strategic spatial attention in parietal cortex. Neuron 16:925-930.

Chudasama Y, Passetti F, Rhodes SEV, Lopian D, Desai A, Robbins TW (2003) Dissociable aspects of performance on the 5-choice serial reaction time task following lesions of the dorsal anterior cingulate, infralimbic and orbitofrontal cortex in the rat: differential effects on selectivity, impulsivity and compulsivity. Behav Brain Res 146:105-119.

Coull J (1998) Neural correlates of attention and arousal: insights from electrophysiology, functional neuroimaging and psychopharmacology. Prog Neurobiol 55:343-361.

Coull JT, Nobre AC (1998) Where and when to pay attention: the neural systems for directing attention to spatial locations and to time intervals as revealed by both PET and fMRI. J Neurosci 18:7426-7435.

Dalley JW, McGaughy J, O'Connell MT, Cardinal RN, Levita L, Robbins TW (2001) Distinct changes in cortical acetylcholine and noradrenaline efflux during contingent and non-contingent performance of a visual attentional task. J Neurosci 21:4908-4914.

Dalley JW, Cardinal RN, Robbins TW (2004) Prefrontal executive functions in rodents: neural and neurochemical substrates. Neurosci Biobehav Rev 28:771-784.

Dias R, Robbins T, Roberts A (1997) Dissociable forms of inhibitory control within prefrontal cortex with an analog of the Wisconsin card sort test: restriction to novel situations and independence from "on-line" processing. J Neurosci 17:9285-9297.

Gill TM, Sarter M, Givens B (2000) Sustained visual attention performanceassociated prefrontal neuronal activity evidence for cholinergic modulation. J Neurosci 20:4745-4757.

Hasselmo M, Bower JM (1992) Cholinergic suppression specific to intrinsic not afferent fiber synapses in rat piriform (olfactory) cortex. J Neurophysiol 67:1222-1229.

Hasselmo M, McGaughy J (2004) High acetylcholine sets circuit dynamics for attention and encoding and low acetylcholine sets dynamics for consolidation. Prog Brain Res 145:207-231.

Hasselmo ME (1999) Neuromodulation: acetylcholine and memory consolidation. Trends Cogn Sci 3:351-359.

Himmelheber A, Sarter M, Bruno J (2000) Increases in cortical acetylcholine release during sustained attention performance in rats. Cognit Brain Res 9:313-325.

Kozak R, Bruno JP, Sarter M (2006) Augmented prefrontal acetylcholine release during challenged attentional performance. Cereb Cortex 16:9-17.

Maddux J-M, Kerfoot EC, Chatterjee S, Holland PC (2007) Dissociation of attention in learning and action: effects of lesions of the amygdala central nucleus, medial prefrontal cortex, and posterior parietal cortex. Behav Neurosci 121:63-79.

McGaughy J, Sarter M (1995a) Behavioral vigilance in rats: task validation and effects of age, amphetamine and benzodiazepine receptor ligands. Psychopharmacology 117:340-357.

McGaughy J, Sarter M (1995b) Effects of chlordiazepoxide and scopolamine but not aging on the detection and identification of conditional visual stimuli. J Gerontol Biol Sci 50:B90-B96.

McGaughy J, Sarter M (1998) Sustained attention performance in rats with intracortical infusions of 192 IgG-saporin-induced cortical cholinergic deafferentation: effects of physostigmine and FG 7142. Behav Neurosci 112:1519-1525.

McGaughy J, Sarter M (1999) Effects of ovariectomy, 192 IgG-saporininduced cortical, cholinergic deafferentation, and administration of estradiol on sustained attention performance in rats. Behav Neurosci 113:1216-1232.

McGaughy J, Kaiser T, Sarter M (1996) Behavioral vigilance following infusions of 192 IgG-saporin into the basal forebrain: selectivity of the behavioral impairment and relation to cortical AChE-positive fiber density. Behav Neurosci 110:247-265.

McGaughy J, Decker MW, Sarter M (1999) Enhancement of sustained attention performance by the nicotinic receptor agonist ABT-418 in intact but not basal forebrain-lesioned rats. Psychopharmacology 144:175-182.

McGaughy J, Everitt BJ, Robbins TW, Sarter M (2000) The role of cortical cholinergic afferent projections in cognition: impact of new selective immunotoxins. Behav Brain Res 115:251-263.

McGaughy J, Dalley JW, Morrison CH, Everitt BJ, Robbins TW (2002a) Selective behavioral and neurochemical effects of cholinergic lesions produced by intrabasalis infusions of $192 \mathrm{IgG}$-saporin on attentional performance in a 5 choice serial reaction time task. J Neurosci 22:1905-1913.

McGaughy JA, Rubin S, Stollstorff M, Baxter MG, Eichenbaum H (2002b) 192 IgG saporin-induced cortical cholinergic deafferentation in rats produces a dissociation in the function of prelimbic/infralimbic and orbitofrontal cortex in an attentional set shifting task. Soc Neurosci Abstr 28:674.4.

McGaughy J, Koene RA, Eichenbaum H, Hasselmo ME (2005) Cholinergic deafferentation of the entorhinal cortex in rats impairs encoding of novel but not familiar stimuli in a delayed nonmatch-to-sample task. J Neurosci 25:10273-10281.

Mesulam M (1999) Spatial attention and neglect: parietal, frontal and cingulate contributions to the mental representation and attentional targeting of salient extrapersonal events. Philos Trans R Soc Lond B Biol Sci 354:1325-1346.

Mesulam M, Mufson EJ, Levey A, Wainer B (1983) Central cholinergic pathways in the rat: an overview based on alternative nomenclature (Ch1Ch6). Neuroscience 10:1185-1201.

Muir JL, Everitt BJ, Robbins TW (1994) AMPA-induced excitotoxic lesions of the basal forebrain: a significant role for the cortical cholinergic system in attentional function. J Neurosci 14:2313-2326.

Muir JL, Everitt BJ, Robbins TW (1995) Reversal of visual attentional dysfunction following lesions of the cholinergic basal forebrain by physostigmine and nicotine and not by the 5-HT3 receptor antagonist, ondansetron. Psychopharmacology 118:82-92.

Nelson CL, Sarter M, Bruno JP (2005) Prefrontal cortical modulation of acetylcholine release in posterior parietal cortex. Neuroscience 132:347-359.

Parasuraman R, Warm JS, Dember WN (1987) Vigilance: Taxonomy and 
utility. In: Ergonomics and human factors (Mark LS, Warm JS, Huston RL, ed), pp 11-32. New York: Springer.

Posner MI, Walker JA, Friedrich FA, Rafal RD (1987) How do the parietal lobes direct cover attention? Neuropsychology 25:135-145.

Ross RS, McGaughy J, Eichenbaum H (2005) Acetylcholine in the orbitofrontal cortex is necessary for the acquisition of a socially transmitted food preference. Learn Mem 12:302-306.

Sarter M, Bruno JP (1997) Cognitive functions of cortical acetylcholine: toward a unifying hypothesis. Brain Res Rev 23:28-46.

Sarter M, Bruno JP (1999) Abnormal regulation of corticopetal cholinergic neurons and impaired information processing in neuropsychiatric disorders. Trends Neurosci 22:67-74.

Sarter M, Bruno JP, Turchi J (1999) Basal forebrain afferent projections modulation cortical acetylcholine, attention, and implications for neuropsychiatric disorders. Ann NY Acad Sci 877:368-382.

Sarter M, Givens B, Bruno JP (2001a) The cognitive neuroscience of sustained attention: where top-down meets bottom-up. Brain Res Rev 35:146-160.

Sarter M, Bruno JP, Berntson GG (2001b) Psychotogenic properties of benzodiazepine receptor inverse agonists. Psychopharmacology 156:1-13.

Sarter M, Hasselmo ME, Bruno JP, Givens B (2005) Unraveling the atten- tional functions of cortical cholinergic inputs: interactions between signal-driven and cognitive modulation of signal detection. Behav Brain Res Rev 48:98-111.

Scerbo MW, Warm JS, Doettling VS, Parasuraman R, Fisk AD (1987) Event asynchrony and task demands in sustained attention. In: Ergonomics and human factors: recent research. (Mark LS, Warm JS, Huston RL, eds), pp 33-39. New York: Springer.

Shomstein S, Yantis S (2004) Control of attention shifts between vision and audition in human cortex. J Neurosci 24:10702-10706.

Shomstein S, Yantis S (2006) Parietal cortex mediates voluntary control of spatial and nonspatial auditory attention. J Neurosci 26:435-439.

Tago H, Kimura H, Maeda T (1986) Visualization of detailed acetylcholinesterase fiber and neuron staining in rat brain by a sensitive histochemical procedure. J Histochem Cytochem 34:1431-1438.

Turchi J, Sarter M (1997) Cortical acetylcholine and processing capacity: effects of cortical cholinergic deafferentation on crossmodal divided attention in rats. Cognit Brain Res 6:147-158.

Yu A, Dayan P (2002) Acetylcholine in cortical inference. Neural Netw 15:719-730.

Yu A, Dayan P (2005) Uncertainty, neuromodulation and attention. Neuron 46:681-692. 